UDK 581.543:630.181

DOI 10.37279/2413-1725-2021-7-2-80-86

\title{
PHYTOMELIORATIVE ROLE OF SHRUB BELT IN ROADSIDE PLANTATIONS
}

\author{
Ivanisova N. V. ${ }^{1}$, Kurinskaya L. V. ${ }^{1}$, Kolesnikov S. I. ${ }^{2}$, Davydenko N. M. ${ }^{1}$ \\ ${ }^{1}$ Novocherkassk Engineering and Land Reclamation Institute named after A. K. Kortunov, Donskoy \\ State Agrarian University, Novocherkassk, Russia \\ ${ }^{2}$ Academy of Biology and Biotechnology, Southern Federal University, Rostov-on-Don, Russia \\ E-mail: lyubov.kurinskay@mail.ru
}

\begin{abstract}
Along the federal roads of the Rostov region, Krasnodar Territory and the Stavropol Territory, it is increasingly possible to find clean plantations consisting of one or two tree species, with a completely destroyed bush belt. In $62 \%$ of the plots studied, the shrub belt is represented by species of scumpia (Cotinus coggygria, $17 \%$ ), terna (Prunus spinosa, $9 \%$ ), elm (Ulmus parvifolia, $5 \%$ ), maple (Acer tataricum, $3 \%$ ), that is, resistant to anthropogenic and climatic factors steppe The bush belt in protective roadside strips plays a significant role in the accumulation of soil moisture (by $6.3 \%$ ), humus (by $2.4 \%$ ), in reducing the noise load (by $25 \mathrm{~dB}$ ). Ancestral and overband shrubs make it possible to create a dense structure of forest strips, which fully performs protective functions both from drifts of the highway bed with snow, and to reduce the negative impact of vehicles on adjacent landscapes. The study of the phytomeliorative role of the shrub belt in existing roadside plantations is a relevant area of research for the further development of recommendations for the creation and maintenance of protective roadside plantings in the steppe zone of southern Russia. Keywords: shrub, phytomeliorative role, soils, roadside plantations, noise, illumination.
\end{abstract}

\section{INTRODUCTION}

Roadside protective plantations in the steppe zone of southern Russia play a significant role in the regulation of wind flows, the distribution of heat, moisture and pollutants. Many years of experience of steppe forestry has proved that plantations should be multicultural, consisting of the main and accompanying breeds and the shrub belt [1]. The bush belt is a component of protective forest stripes located either up to the main forest stripes or after. It prevents the penetration of steppe elements under the canopy of plantations.

To create sustainable plantations, it is necessary to take into account the natural and climatic conditions, functional purpose and environmental and biological characteristics of each species. Shrub belt in protective plantations performs soil protective role, reduces evaporation of moisture, prevents blowing [2], reduces overgrowth by weed vegetation, reduces noise pollution [3]. But recently, there has been a tendency when creating or caring for protective plantations not to design a bush belt, but to introduce or leave only the main or accompanying breed. Along the federal roads of the Rostov region, Krasnodar and Stavropol Territory, clean plantations consisting of one or two tree species with a 
completely destroyed bush belt are increasingly found. The study of the reclamation role of the shrub belt in existing roadside plantations is a relevant area of research for the further development of recommendations on the creation and maintenance of protective roadside plantations in the steppe zone of southern Russia [4].

\section{MATERIALS AND METHODS}

The research was carried out in the period 2017-2019, two motor roads were chosen as the research targets: of the federal (M4 Novocherkassk-Rostov) and regional significance (Novocherkassk-Shakhty), experiencing various anthropogenic impacts (fig. 1).

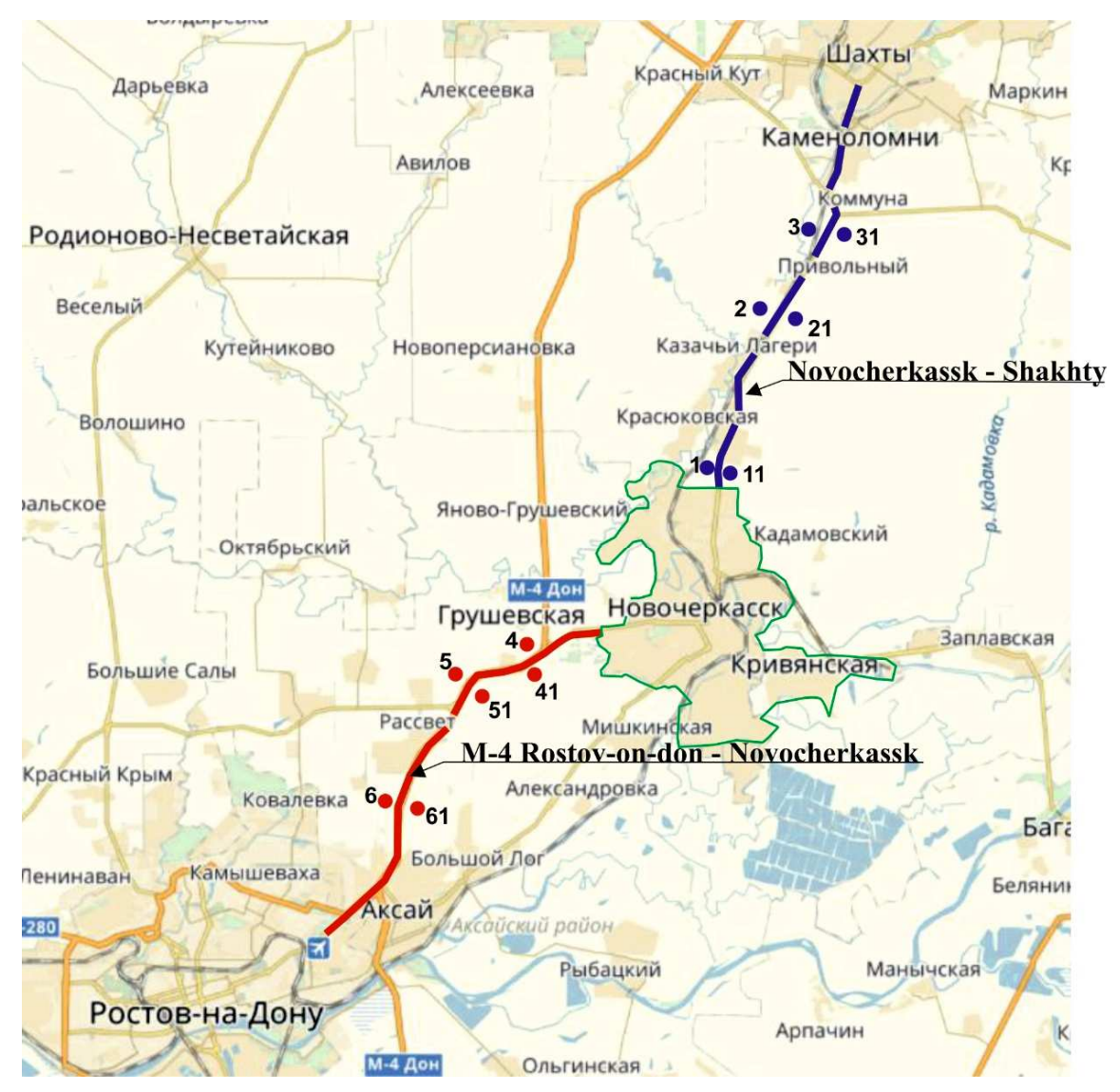

Fig.1. Map - scheme of location of objects of research.

The distance from the roadbed edge to the forest growing stock was considered when laying the test areas (TA). The sampling area was located at a distance directly near the road, 10-100 $\mathrm{m}$ from the growing stock and under the growing stock, in the zones of the so-called "edge" effect of the impact of vehicles on ecosystems and especially on biota.

The assessment of the protective roadside growing stock state is given in accordance with the tree state category scale (Sanitary rules in forests of the Russian Federation, 2013). 
Single samples were taken on the TA from the upper soil horizon by the envelope method. PH of water extract (GOST 26423-85), humus determination by I. V. Tyurin method (GOST 26213-91) were determined in soil samples. The assessment of the noise protective role of the shrub zone was carried out in the state with leaves and without them using the Testo 816-4 sound level meter. The measurements were carried out in front of, in and behind growing stock directly from the noise source [5]. Measurement of luminosity in growing stock with participation of a shrub zone and without it was carried out by means of the TKA-Lux luxmeter.

\section{RESULTS AND DISCUSSION}

According to the current recommendations, the roadside forest strips in the steppe zone are formed by means of a shrub zone. Which is located either immediately after the road ditch part, or after the protective strips on the side of adjacent agricultural fields [6]. The main types of shrub vegetation and their ecological and biological features are presented in table 1. Considering climatic and anthropogenic factors, the stability of shrubs in the system of roadside growing stock was assessed separately.

Table 1

Ecological and biological features of the roadside growing stock shrubs of the steppe zone

\begin{tabular}{|c|c|c|c|c|c|}
\hline $\mathbf{N o}$ & Sort & $\begin{array}{c}\text { Relationship to } \\
\text { illumination }\end{array}$ & Relation to soil & $\begin{array}{c}\text { Relation to } \\
\text { moisture }\end{array}$ & Stability \\
\hline $\mathbf{1}$ & $\mathbf{2}$ & $\mathbf{3}$ & $\mathbf{4}$ & $\mathbf{5}$ & $\mathbf{6}$ \\
\hline 1 & $\begin{array}{c}\text { Amórpha } \\
\text { fruticósa }\end{array}$ & light-loving. & undemanding & undemanding & $\begin{array}{c}3 \text { (not } \\
\text { resistant to } \\
\text { salinization) }\end{array}$ \\
\hline 2 & $\begin{array}{c}\text { Ulmus } \\
\text { parvifolia }\end{array}$ & light-loving & exacting & drought-resistant & 3 \\
\hline 3 & $\begin{array}{c}\text { Cotinus } \\
\text { coggýgria }\end{array}$ & shade-tolerant & undemanding & drought-resistant & 1 \\
\hline 4 & $\begin{array}{c}\text { Acer } \\
\text { tatáricum }\end{array}$ & shade-tolerant & undemanding & drought-resistant & 1 \\
\hline 5 & $\begin{array}{c}\text { Caragána } \\
\text { arboréscens }\end{array}$ & light-loving & undemanding & undemanding & 1 \\
\hline 6 & $\begin{array}{c}\text { Ligústrum } \\
\text { vulgáre }\end{array}$ & shade-tolerant & those with low & drought-resistant & 1 \\
\hline 7 & $\begin{array}{c}\text { Lonícera } \\
\text { tatárica }\end{array}$ & shade-tolerant & undemanding & drought-resistant & 1 \\
\hline 8 & $\begin{array}{c}\text { Elaeágnus } \\
\text { angustifólia }\end{array}$ & light-loving & undemanding & drought-resistant & 2 \\
\hline 9 & $\begin{array}{c}\text { Ribes } \\
\text { aureum }\end{array}$ & shade-tolerant & undemanding & drought-resistant & 1 \\
\hline
\end{tabular}


Continuation of table 1

\begin{tabular}{|c|c|c|c|c|c|}
\hline 10 & Tamarix & shade-tolerant & undemanding & drought-resistant & 2 \\
\hline 11 & $\begin{array}{c}\text { Prunus } \\
\text { spinosa }\end{array}$ & light-loving & undemanding & drought-resistant & 1 \\
\hline 12 & $\begin{array}{c}\text { Rosea } \\
\text { cinnamomea }\end{array}$ & light-loving & undemanding & drought-resistant & 1 \\
\hline 13 & Hippophae & light-loving & undemanding & exacting & 2 \\
\hline 14 & $\begin{array}{c}\text { Córylus } \\
\text { avellána }\end{array}$ & shade-tolerant & undemanding & drought-resistant & 3 \\
\hline 16 & $\begin{array}{c}\text { Córnus mas } \\
\text { Amelanchier }\end{array}$ & shade-tolerant & exacting & drought-resistant & $\begin{array}{c}3 \text { (not } \\
\text { resistant to } \\
\text { dry winds) }\end{array}$ \\
\hline 17 & $\begin{array}{c}\text { Swida } \\
\text { sanguinea }\end{array}$ & shade-tolerant & undemanding & drought-resistant & 1 \\
\hline
\end{tabular}

Stability category: 1 - stable; 2 - medium-stable; 3 - unstable for abiotic and biotic factors; 4 based on anthropogenic factors.

Taxation description of roadside protective plantings at the studied objects is given in Table 2. The predominant breed in protective plantations is Robinia pseudoacacia and Fraxinus lanceolata. In multi-row bands, all other things being equal, the life activity of trees and shrubs is influenced by their spatial placement and breed composition, which determine the degree of competitive relations between them [5].

Taxing characteristic of roadside protective plantations

Table 2

\begin{tabular}{|c|c|c|c|c|c|c|}
\hline No & Location & The predominant sort & $\begin{array}{c}\text { Number } \\
\text { of rows } \\
\text { in the } \\
\text { lane }\end{array}$ & $\begin{array}{c}\text { The edges } \\
\text { line up } \\
\text { with the } \\
\text { side of the } \\
\text { road }\end{array}$ & $\begin{array}{c}\text { Shrub belt } \\
\text { inside } \\
\text { plantings }\end{array}$ & $\begin{array}{c}\text { Edge rows } \\
\text { on the side } \\
\text { of } \\
\text { agricultural } \\
\text { fields }\end{array}$ \\
\hline \multicolumn{7}{|c|}{ Novocherkassk- Shakhty } \\
\hline 1 & $\begin{array}{c}50 \mathrm{~m} \text { from } \\
\text { the road }\end{array}$ & $\begin{array}{c}50 \% \text { Robinia pseudoacácia } \\
\text { and 50\% Fraxinus lanceolata }\end{array}$ & 6 & - & $\begin{array}{c}\text { Ulmus } \\
\text { parvifolia }\end{array}$ & $\begin{array}{c}\text { Prunus } \\
\text { spinosa }\end{array}$ \\
\hline 1.1 & $\begin{array}{c}20 \mathrm{~m} \text { from } \\
\text { the road }\end{array}$ & $\begin{array}{c}50 \% \text { Robínia pseudoacácia } \\
\text { and 50\% Fraxinus lanceolata }\end{array}$ & 6 & - & $\begin{array}{c}\text { Ulmus } \\
\text { parvifolia }\end{array}$ & - \\
\hline 2 & $\begin{array}{c}50 \mathrm{~m} \text { from } \\
\text { the road }\end{array}$ & $\begin{array}{c}50 \% \text { Robinia pseudoacácia } \\
\text { and 50\% Ácer negúndo }\end{array}$ & 6 & $\begin{array}{c}\text { Cotinus } \\
\text { coggýgria }\end{array}$ & - & $\begin{array}{c}\text { Elaeágnus } \\
\text { angustifólia }\end{array}$ \\
\hline 2.1 & $\begin{array}{c}20 \mathrm{~m} \text { from } \\
\text { the road }\end{array}$ & $\begin{array}{c}50 \% \text { Robinia pseudoacácia } \\
\text { and 50\% Fraxinus lanceolata }\end{array}$ & 6 & $\begin{array}{c}\text { Cotinus } \\
\text { coggýgria }\end{array}$ & $\begin{array}{c}\text { Ulmus } \\
\text { parvifolia }\end{array}$ & - \\
\hline 3 & $\begin{array}{c}20 \mathrm{~m} \text { from } \\
\text { the road }\end{array}$ & $\begin{array}{c}50 \% \text { Robinia pseudoacácia } \\
\text { and 50\% Fraxinus lanceolata }\end{array}$ & 6 & $\begin{array}{c}\text { Cotinus } \\
\text { coggýgria }\end{array}$ & $\begin{array}{c}\text { Ácer } \\
\text { tatáricum }\end{array}$ & $\begin{array}{c}\text { Prunus } \\
\text { spinosa }\end{array}$ \\
\hline
\end{tabular}


Continuation of table 2

\begin{tabular}{|c|c|c|c|c|c|c|}
\hline 3.1 & $\begin{array}{l}50 \mathrm{~m} \text { from } \\
\text { the road }\end{array}$ & $\begin{array}{l}50 \% \text { Robínia pseudoacácia } \\
\text { and } 50 \% \text { Fraxinus lanceolata }\end{array}$ & 6 & $\begin{array}{c}\text { Cotinus } \\
\text { coggýgria }\end{array}$ & $\begin{array}{c}\text { Ácer } \\
\text { tatáricum }\end{array}$ & - \\
\hline \multicolumn{7}{|c|}{ Rostov-on-Don - Novocherkassk } \\
\hline 4 & $\begin{array}{l}20 \mathrm{~m} \text { from } \\
\text { the road }\end{array}$ & $\begin{array}{c}80 \% \text { Robínia pseudoacácia } \\
\text { and } 20 \% \text { Quércus róbur }\end{array}$ & 5 & - & $\begin{array}{c}\text { Swida } \\
\text { sanguinea }\end{array}$ & - \\
\hline 4.1 & $\begin{array}{l}20 \mathrm{~m} \text { from } \\
\text { the road }\end{array}$ & $\begin{array}{c}50 \% \text { Robínia pseudoacácia } \\
\text { and } 50 \% \text { Fraxinus } \\
\text { lanceolata }\end{array}$ & 6 & $\begin{array}{l}\text { Cotinus } \\
\text { coggýgria, } \\
\text { Swida } \\
\text { sanguinea }\end{array}$ & - & $\begin{array}{l}\text { Prunus } \\
\text { spinosa }\end{array}$ \\
\hline 5 & $\begin{array}{l}50 \mathrm{~m} \text { from } \\
\text { the road }\end{array}$ & $\begin{array}{c}60 \% \text { Robínia pseudoacácia } \\
\text { and } 30 \% \text { Fraxinus } \\
\text { lanceolate and } 10 \% \\
\text { Quércus róbur }\end{array}$ & 7 & $\begin{array}{l}\text { Cotinus } \\
\text { coggýgria, } \\
\text { Swida } \\
\text { sanguinea } \\
\end{array}$ & - & $\begin{array}{l}\text { Prunus } \\
\text { spinosa }\end{array}$ \\
\hline 5.1 & $\begin{array}{l}20 \mathrm{~m} \text { from } \\
\text { the road }\end{array}$ & $\begin{array}{c}50 \% \text { Fraxinus lanceolata } \\
\text { and } 50 \% \text { Robínia } \\
\text { pseudoacácia }\end{array}$ & 6 & $\begin{array}{l}\text { Cotinus } \\
\text { coggýgria, } \\
\text { Swida } \\
\text { sanguinea }\end{array}$ & $\begin{array}{c}\text { Swida } \\
\text { sanguinea }\end{array}$ & - \\
\hline 6 & $\begin{array}{l}50 \mathrm{~m} \text { from } \\
\text { the road }\end{array}$ & $\begin{array}{l}60 \% \text { Fraxinus lanceolata } \\
\text { and } 20 \% \text { Populus } \\
\text { pyramidalis, } 10 \% \text { Quércus } \\
\text { róbur, } 10 \% \text { Ácer negúndo }\end{array}$ & 7 & $\begin{array}{l}\text { Cotinus } \\
\text { coggýgria, } \\
\text { Swida } \\
\text { sanguinea } \\
\end{array}$ & - & $\begin{array}{c}\text { Rosea } \\
\text { cinnamome } \\
\text { a }\end{array}$ \\
\hline 6.1 & $\begin{array}{l}10 \mathrm{~m} \text { from } \\
\text { the road }\end{array}$ & $100 \%$ Fraxinus lanceolata & 1 & - & - & - \\
\hline
\end{tabular}

The highest percentage (more than $62 \%$ ) in the studied roadside growing stock is occupied by the shrub zone consisting of Cotinus coggýgria, $17 \%$ - Prunus spinosa, $9 \%$ - Ulmus parvifolia, $5 \%$ - Ácer tatáricum, i.e. from species resistant to both anthropogenic and climatic factors of the steppe zone

When studying the soil protection role of the shrub zone, special attention was paid to such edaphic factors as $\mathrm{pH}$, humus content, aggregate composition, soil moisture (table 3).

On the test area without a shrub zone, there is a decrease in the content of humus, soil compaction and a decrease in humidity. In the presence of a shrub in the edge tree rows and inside the growing stock, soil moisture and humus content is increased. It is also noted that in the test areas with the shrub zone, the middle loamy neutral soils predominate and moisture evaporation decreases.

The presence of the shrub zone up to $1.5 \mathrm{~m}$ in height significantly increases the noise absorbing capacity of roadside forest strips (table 4). But the greatest noise absorbing effect of the shrub tier is observed from the roadbed.

For effective dispersion of sunlight in roadside forest strips, its width should be more than 30 meters with several shrub species both in the edge tree rows on the roadbed side and inside the growing stock (table 4). 
Table 3

Influence of the shrub zone on the roadside landscapes soils

\begin{tabular}{|c|l|c|c|c|c|}
\hline № & \multicolumn{1}{|c|}{ Shrubby belt } & $\mathbf{p H}$ & $\begin{array}{c}\text { Content of } \\
\text { humus, \% }\end{array}$ & $\begin{array}{c}\text { Aggregate } \\
\text { content } \mathbf{\%}): \\
<\mathbf{0 . 2 5} \mathbf{~ m m}\end{array}$ & $\begin{array}{c}\text { Humidity, } \\
\mathbf{\%}\end{array}$ \\
\hline 1 & $\begin{array}{l}\text { inside plantings and } \\
\text { beyond the band belt }\end{array}$ & $7,80 \pm 0,06$ & $5,80 \pm 0,06$ & $0,60 \pm 0,07$ & $4,69 \pm 0,08$ \\
\hline 1.1 & inside plantings & $7,60 \pm 0,11$ & $6,66 \pm 0,06$ & $0,16 \pm 0,02$ & $6,17 \pm 0,37$ \\
\hline 2 & forest edge number & $7,30 \pm 0,11$ & $5,66 \pm 0,06$ & $0,46 \pm 0,05$ & $5,50 \pm 0,41$ \\
\hline 2.1 & $\begin{array}{l}\text { forest edge number } \\
\text { and inside plantings }\end{array}$ & $7,90 \pm 0,11$ & $4,76 \pm 0,03$ & $0,80 \pm 0,06$ & $5,35 \pm 0,36$ \\
\hline 3 & $\begin{array}{l}\text { forest edge number } \\
\text { and inside plantings }\end{array}$ & $7,80 \pm 0,11$ & $6,82 \pm 0,07$ & $0,50 \pm 0,11$ & $5,11 \pm 0,22$ \\
\hline 3.1 & $\begin{array}{l}\text { forest edge number } \\
\text { and inside plantings }\end{array}$ & $7,50 \pm 0,11$ & $5,00 \pm 0,13$ & $0,56 \pm 0,02$ & $4,69 \pm 0,61$ \\
\hline 4 & forest edge number & $7,70 \pm 0,17$ & $6,05 \pm 0,09$ & $0,16 \pm 0,01$ & $6,86 \pm 1,36$ \\
\hline 4.1 & forest edge number & $7,60 \pm 0,06$ & $8,30 \pm 0,14$ & $0,40 \pm 0,13$ & $6,73 \pm 0,27$ \\
\hline 5 & forest edge number & $7,80 \pm 0,09$ & $5,02 \pm 0,05$ & $0,27 \pm 0,01$ & $6,12 \pm 0,61$ \\
\hline 5.1 & $\begin{array}{l}\text { forest edge number } \\
\text { and inside plantings }\end{array}$ & $7,70 \pm 0,06$ & $5,76 \pm 0,12$ & $0,20 \pm 0,08$ & $6,87 \pm 0,11$ \\
\hline 6 & forest edge number & $7,80 \pm 0,11$ & $3,83 \pm 0,07$ & $0,83 \pm 0,02$ & $8,67 \pm 0,09$ \\
\hline 6.1 & $\begin{array}{l}\text { control (inside } \\
\text { plantings) }\end{array}$ & $7,60 \pm 0,06$ & $2,66 \pm 0,07$ & $0,10 \pm 0,03$ & $3,94 \pm 1,04$ \\
\hline
\end{tabular}

Influence of shrubs on noise pollution and illumination

Table 4

\begin{tabular}{|c|c|c|c|c|c|c|}
\hline \multirow{2}{*}{ № } & \multicolumn{2}{|c|}{ Noise pollution, decibel } & \multicolumn{3}{c|}{ Illumination, Lux } \\
\cline { 2 - 7 } & before & inside & behind & before & inside & behind \\
\hline 1 & $74 \pm 3,02$ & $59 \pm 2,0$ & $50 \pm 3,39$ & $16,8 \pm 0,25$ & $2,5 \pm 0,47$ & $7,8 \pm 0,80$ \\
\hline 1.1 & $82 \pm 1,58$ & $70 \pm 3,81$ & $63 \pm 4,80$ & $9,2 \pm 0,52$ & $5,6 \pm 0,27$ & $11,3 \pm 0,20$ \\
\hline 2 & $78 \pm 2,09$ & $63 \pm 2,65$ & $60 \pm 2,00$ & $19,5 \pm 0,33$ & $4,6 \pm 0,33$ & $6,7 \pm 0,39$ \\
\hline 2.1 & $80 \pm 1,87$ & $75 \pm 1,58$ & $75 \pm 1,58$ & $10,3 \pm 0,22$ & $2 \pm 0,32$ & $18,3 \pm 0,72$ \\
\hline 3 & $60 \pm 3,32$ & $55 \pm 2,92$ & $53 \pm 4,80$ & $3,5 \pm 0,46$ & $3,0 \pm 0,45$ & $7,4 \pm 0,23$ \\
\hline 3.1 & $78 \pm 1,58$ & $67 \pm 4,42$ & $60 \pm 2,92$ & $7,3 \pm 0,27$ & $6 \pm 0,33$ & $15,1 \pm 0,22$ \\
\hline 4 & $80 \pm 3,87$ & $32 \pm 2,35$ & $70 \pm 1,41$ & $68,6 \pm 0,71$ & $2,6 \pm 0,27$ & $19,2 \pm 0,48$ \\
\hline 4.1 & $90 \pm 2,45$ & $45 \pm 2,92$ & $60 \pm 2,00$ & $76,0 \pm 1,51$ & $0,08 \pm 0,03$ & $70,0 \pm 0,73$ \\
\hline 5 & $95 \pm 5,34$ & $50 \pm 1,22$ & $60 \pm 3,74$ & $66,0 \pm 2,12$ & $2,6 \pm 0,47$ & $66,5 \pm 0,49$ \\
\hline 5.1 & $90 \pm 3,46$ & $55 \pm 1,58$ & $95 \pm 2,24$ & $31 \pm 2,55$ & $0,03 \pm 0,02$ & $32,0 \pm 0,47$ \\
\hline 6 & $80 \pm 1,41$ & $50 \pm 2,55$ & $70 \pm 2,70$ & $31,4 \pm 1,20$ & $18,5 \pm 0,37$ & $32,0 \pm 1,10$ \\
\hline 6.1 & $80 \pm 6,20$ & $75 \pm 1,00$ & $55 \pm 2,24$ & $27,4 \pm 0,44$ & $30,6 \pm 1,12$ & $35,6 \pm 2,08$ \\
\hline
\end{tabular}




\section{CONCLUSIONS}

The presence of two or more shrub rows in the edge tree rows of protective growing stock allows to reduce moisture evaporation, over-compaction and acidification of the upper soil horizon.

Roadside forest strips of dense impermeable construction with the shrub species allow to achieve maximum noise reduction from the motor road, to reduce luminosity at frontal and lateral lighting of the roadbed.

The introduction of two or more shrub species into the system of protective growing stock will allow to create a sustainable phyto-meliorative phytocoenosis.

The work was supported by the State Program for Leading Scientific Schools of the Russian Federation (NSH-2511.2020.11)

\section{References}

1. Zdornov I. A., Nagimov Z. Ya., Kapralov A. V. Sanitary condition of roadside protective forest lanes in northern Kazakhstan, Successes of modern natural science, 3, 44 (2018).

2. Chernikov E. A., Goptarev S. M., Morkovin V. A. Influence of protective forest strips on the ecological state of the roadside territory, Voronezh Scientific and Technical Bulletin, 3 (21), 74 (Voronezh, 2017).

3. Ivanova A. N. The influence of the parameters of roadside forest lanes on reducing noise near roads (using the example of the Saratov right bank): diss.... edging. biol. Sciences, 118. (Saratov, 2014).

4. Kokin A. V., Shumakova G. E. Agroecobsecurity of timber and open agroecosystems of the South of Russia, Bulletin MANEB, 20, 1, 116 (2015).

5. Ivanisova N. V., Davydenko N. M., Kurinskaya L. V., Kolesnikov S. I. The protective effect of plantations on soil of roadsive landscapes, 18th International Multidisciplinary Scientific GeoConference SGEM 2018, 635 (CONFERENCE PROCEEDINGS, 2018).

6. Bechina D. N., Boytsova E. N. The influence of wood-shrub vegetation on noise reduction on the example of Saratov, Environmental and technological aspects of forestry in the steppe and forest-steppe: materials conf, Ed. A. V. Golubev; FGOU VPO "Saratov GAU", 108 p. (Saratov, 2007).

Иванисова Н. В. Фитомелиоративная роль кустарникового пояса в придорожных насаждениях / Н. В. Иванисова, Л. В. Куринская, С. И. Колесников, Н. М. Давыденко // Ученые записки Крымского федерального университета им. В. И. Вернадского. Биология, химия. - 2021. - Т. 7 (73), №2 . - С. 80-86. Вдоль федеральных дорог Ростовской области, Краснодарского края и Ставропольского края все чаще можно встретить чистые насаждения, состоящие из одной или двух древесных пород, с полностью уничтоженным кустарниковым поясом. В $62 \%$ исследованных насаждений кустарниковый пояс представлен видами скумпии (Cotinus coggygria, 17 \%), терна (Prunus spinosa, 9 \%), вяза (Ulmus parvifolia, $5 \%$ ), клена (Acer tataricum, $3 \%$ ), есть устойчивыми к антропогенным и климатическим факторам степной зоны. Кустарниковый пояс в защитных придорожных полосах играет существенную роль в накоплении почвенной влаги (на 6,3 \%), гумуса (на 2,4 \%), в снижении шумовой нагрузки (на 25 дБ). Прикюветные и заполосные кустарниковые насаждения позволяют создать плотную конструкцию лесных полос, которая в полной мере выполняет защитные функции как от заносов полотна автодороги снегом, так и по снижению негативного влияния автотранспорта на сопредельные ландшафты. Изучение фитомелиоративной роли кустарникового пояса в существующих придорожных насаждениях является актуальным направлением исследований для дальнейшей разработки рекомендаций по созданию и содержанию защитных придорожных насаждений в степной зоне юга России.

Ключевые слова: кустарник, фитомелиоративная роль, почвы, придорожные насаждения, шум, освещенность. 